\title{
Reply To Kenneth B. Yatai, Mark J. Dunning, Dennis Wang. Consensus Genomic Subtypes of Muscle-invasive Bladder Cancer: A Step in the Right Direction but Still a Long Way To Go. Eur Urol 2020;77:434-5
}

\author{
Aurélie Kamoun ${ }^{a, *}$, Aurélien de Reyniès $^{b}$, Yves Allory $^{c}$, Gottfrid Sjödahl $^{d}$, A. Gordon Robertson $^{e}$, Roland Seiler $^{f}$, \\ Katherine A. Hoadley ${ }^{g}$, Clarice S. Groeneveld ${ }^{h}$, Hikmat Al-Ahmadie ${ }^{i}$, Woonyoung Choi ${ }^{j}$, Mauro A.A. Castro ${ }^{k}$, \\ Jacqueline Fontugne ${ }^{c}$, Pontus Eriksson ${ }^{l}$, Qianxing Mo $^{m}$, Jordan Kardos ${ }^{g}$, Alexandre Zlotta ${ }^{n}$, Arndt Hartmann ${ }^{o}$, \\ Colin P Dinney ${ }^{p}$, Joaquim Bellmunt ${ }^{q}$, Thomas Powles ${ }^{r}$, Núria Malats ${ }^{s}$, Keith S Chan ${ }^{t}$, William Y Kim ${ }^{u}$, David J McConkey ${ }^{j}$, \\ Peter C Black ${ }^{v}$, Lars Dyrskjøt ${ }^{w}$, Mattias Höglund ${ }^{l}$, Seth P Lerner $^{x}$, Francisco X Real ${ }^{y}$, François Radvanyi ${ }^{z}$, Mattias Aine ${ }^{A}$, \\ Isabelle Bernard-Pierrot ${ }^{z}$, Bogdan Czerniak ${ }^{B}$, Ewan A Gibb ${ }^{C}$, Jaegil Kim ${ }^{D}$, David J. Kwiatkowski ${ }^{E}$, Thierry Lebret ${ }^{F}$, \\ Fredrik Liedberg ${ }^{d}$, A Arlene Siefker-Radtke ${ }^{G}$, Nanor Sirab ${ }^{H}$, Ann Taber ${ }^{w}$, John N. Weinstein ${ }^{I}$
}

${ }^{\text {a } C a r t e s ~ d ' I d e n t i t e ́ ~ d e s ~ T u m e u r s ~(C I T) ~ P r o g r a m, ~ L i g u e ~ N a t i o n a l e ~ C o n t r e ~ l e ~ C a n c e r, ~ P a r i s, ~ F r a n c e ; ~}{ }^{\mathrm{b}}$ Cartes d'Identité des Tumeurs (CIT) Program, Ligue Nationale Contre le Cancer, Paris, France; ' Department of Pathology, Institut Curie, Saint-Cloud, France; Institut Curie, PSL Research University, CNRS, UMR144, Equipe Labellisée Ligue Contre le Cancer, Paris, France; ${ }^{\mathrm{d}}$ Division of Urological Research, Department of Translational Medicine, Lund University, Skåne University Hospital, Malmö, Sweden; ${ }^{\mathrm{e} C a n a d a ' s ~ M i c h a e l ~ S m i t h ~ G e n o m e ~ S c i e n c e s ~ C e n t e r, ~ B C ~ C a n c e r ~ A g e n c y, ~ V a n c o u v e r, ~ B C, ~ C a n a d a ; ~}{ }^{\mathrm{f}}$ Department of Urology, Bern University Hospital, Bern, Switzerland; ${ }^{g}$ Department of Genetics, Lineberger Comprehensive Cancer Center, University of North Carolina at Chapel Hill, Chapel Hill, NC, USA; ${ }^{\mathrm{h}}$ Cartes d'Identité des Tumeurs (CIT) Program, Ligue Nationale Contre le Cancer, Paris, France; Institut Curie, PSL Research University, CNRS, UMR144, Equipe Labellisée Ligue Contre le Cancer, Paris, France; Bioinformatics and Systems Biology Laboratory, Federal University of Paraná, Polytechnic Center, Curitiba, Brazil; ${ }^{i}$ Department of Pathology, Memorial Sloan Kettering Cancer Center, New York, NY, USA; ${ }^{\mathrm{j}}$ Johns Hopkins Greenberg Bladder Cancer Institute and Brady Urological Institute, Johns Hopkins University, Baltimore, MD, USA; ${ }^{\mathrm{B}}$ Bioinformatics and Systems Biology Laboratory, Federal University of Paraná, Polytechnic Center, Curitiba, Brazil; ${ }^{1}$ Division of Oncology and Pathology, Department of Clinical Sciences, Lund University, Lund, Sweden; ${ }^{\mathrm{m}}$ Department of Biostatistics and Bioinformatics, H. Lee Moffitt Cancer Center and Research Institute, Tampa, FL, USA; ${ }^{\mathrm{n}}$ Division of Urology, Department of Surgery, University of Toronto, Mount Sinai Hospital and University Health Network, Toronto, ON, Canada; ${ }^{\circ}$ Institute of Pathology, University Erlangen-Nürnberg, Erlangen, Germany; ${ }^{\mathrm{p}}$ Department of Urology, University of Texas MD Anderson Cancer Center, Houston, TX, USA; Department of Cancer Biology, University of Texas MD Anderson Cancer Center, Houston, TX, USA; ${ }^{\mathrm{q}}$ Bladder Cancer Center, DanaFarber/Brigham and Women's Cancer Center, Harvard Medical School, Boston, MA, USA; ${ }^{\mathrm{r}}$ Barts Cancer Institute ECMC, Barts Health and the Royal Free NHS Trust, Queen Mary University of London, London, UK; ${ }^{5}$ Genetic and Molecular Epidemiology Group, Spanish National Cancer Research Centre (CNIO), CIBERONC, Madrid, Spain; ${ }^{\mathrm{t}}$ Cedars-Sinai Samuel Oschin Cancer Institute, Los Angeles, CA, USA; ${ }^{\mathrm{u}}$ Department of Genetics, Lineberger Comprehensive Cancer Center, University of North Carolina at Chapel Hill, Chapel Hill, NC, USA; Department of Medicine, Lineberger Comprehensive Cancer Center, University of North Carolina at Chapel Hill, Chapel Hill, NC, USA; ${ }^{v}$ Department of Urologic Sciences, University of British Columbia, Vancouver, BC, Canada; ${ }^{\mathrm{w}}$ Department of Molecular Medicine, Aarhus University Hospital, Aarhus, Denmark; ${ }^{\mathrm{x}}$ Scott Department of Urology, Dan L. Duncan Cancer Center, Baylor College of Medicine, Houston, TX, USA; ${ }^{\mathrm{y}}$ Epithelial Carcinogenesis Group, Spanish National Cancer Research Centre (CNIO), CIBERONC, Madrid, Spain; ${ }^{\mathrm{Z}}$ Institut Curie, PSL Research University, CNRS, UMR144, Equipe Labellisée Ligue Contre le Cancer, Paris, France; ${ }^{\mathrm{A} D i v i s i o n}$ of Molecular Hematology, Department of Laboratory Medicine, Faculty of Medicine, Lund University, Lund, Sweden; ${ }^{\mathrm{B}}$ Department of Pathology, The University of Texas MD Anderson Cancer Center, Houston, TX, USA; ' GenomeDx Biosciences Inc., Vancouver, BC, Canada; ${ }^{\mathrm{D}}$ Broad Institute of MIT and Harvard, Cambridge, MA, USA; ${ }^{\mathrm{E}}$ Department of Medicine, Brigham and Women's Hospital, Harvard Medical School, Boston, MA, USA; ${ }^{\mathrm{F}}$ Department of Urology, University of Versailles-Saint-Quentin-en-Yvelines, Foch Hospital, Suresnes, France; ${ }^{G}$ Department of Genitourinary Medical Oncology, The University of Texas MD Anderson Cancer Center, Houston, TX, USA; ${ }^{\mathrm{H}}$ Department of Pathology, Institut Curie Hospital Group, Paris, France; ${ }^{\mathrm{I}}$ Department of Bioinformatics and Computational Biology, The University of Texas MD Anderson Cancer Center, Houston, TX, USA

DOI of original article: https://doi.org/10.1016/j.eururo.2019.09.006.

* Corresponding author. Cartes didentité des Tumeurs Program, Ligue Nationale Contre le Cancer, 14 rue Corvisart, 75013 Paris, France.

Tel. +33 153552558 .

E-mail address: aurelie.kamoun@ponts.org (A. Kamoun). 
In our study [1] the Bladder Cancer Molecular Taxonomy Group collaborated to extend a first consensus report [2], addressing the need for a consensus molecular classification for muscle-invasive bladder cancer (MIBC) that would support basic research and clinical trials. We provide such a consensus classification and offer a single-sample classifier (http://cit.ligue-cancer.net:3838/apps/consensusMIBC_web).

Yatai et al [3] acknowledge the need for an MIBC consensus classification and commend our efforts, but suggest limitations in our methodology and data. Our discussion [1] acknowledged limitations related to cohorts, expression assays, and clinical data. Here, we would like to respond to several statements by Yatai et al, as these were at times imprecise, or based on misinterpretation or on results that we reported but were overlooked.

First, we would like to clarify our methodological approach and goals. Using historical transcriptomic data from diverse sources and technologies from 1750 tumors and 18 data sets, we identified a consensus of six published MIBC classifications. Aware of concerns about using data from different microarray platforms, or RNA sequencing (RNA-seq) and microarray data, we did not aggregate the 18 data sets; instead, we ran the six classifiers on each data set independently (Section 2.2, Supplementary Fig. 1 [1]) and then jointly analyzed the six resulting partitions of the 1750 tumors using an approach similar to that described for colorectal cancer [4]. While we emphasized describing the consensus results, we provided classification results for all samples in each of the 18 data sets (Supplementary Fig. 7 [1]). We trained the single-sample classifier on a subset of the microarray [5] and RNA-seq [6] data and then applied it to all remaining samples. We reported performance metrics for the classifier (Supplementary Fig. 9C [1]) and showed that it can be used with both microarray and RNA-seq data. We also tested COCA, which clusters per-sample classification calls (eg, Fig. 6A in [6]); for conciseness, we did not report these results.

Yatai et al state that Guinney's approach [4] "has not always been successful in other cancers", but do not reference this claim. We are aware of two publications that use this consensus subtype approach: the original 2015 work $(n=4151)$ [4], and our work on MIBC $(n=1750)$ [1]. Two other recent studies used different methods to generate single-sample classifiers from subtypes for several separate data sets. Chen et al [7] worked with microarray data from 15 ovarian cancer data sets $(n=1774)$, while Rashid et al [8] worked with microarray and RNA-seq data ( $n=1085$ ) from nine pancreatic cancer data sets. In both studies, classifier features were binary gene pairs for subsets of genes.

Our main goals were to help the development of MIBC precision medicine by providing a robust framework to connect retrospective and prospective clinical findings to molecular contexts, and to identify clinically relevant biomarkers for patient management. Our goals did not include proposing or comparing methods for deriving a consensus from different classification solutions.

Second, we would like to respond to specific criticisms by making several points.
- Bladder cancer is a complex disease, pathologically, clinically, and biologically, and it would be naïve to think that the six consensus classes will capture all of this complexity. Furthermore, any parameter such as treatment response in the presence of a specific mutated gene may need to be tested across subtypes, because the response may vary by subtype.

- The pathological, clinical, and biological characteristics of the consensus subtypes identified are consistent with previous findings, providing additional confidence in our consensus approach. For example, the associations between histological variants were particularly interesting for the squamous variant and the neuroendocrine variant, which were strongly associated with the basal/squamous and the neuroendocrine-like subtypes, respectively.

- While we reported subtype associations for four histological variants (squamous, micropapillary, sarcomatoid, and neuroendocrine), sample sizes prevented us from reporting associations for rarer variants such as plasmacytoid. Future studies are needed to determine whether rarer variants fit within our consensus groups or define additional molecular classes.

- To increase the sample sizes for reporting subtypespecific genetic alterations for seven key bladder-cancer genes, including FGFR3, TP53, and RB1, we used data for copy number, gene fusions, and gene mutations from 600 samples from the 18 cohorts. This allowed us to show that TP53 and RB1 genetic alterations co-occurred in 16 of the 17 neuroendocrine-like tumors that had both sequencing and copy number data (Fig. 3B [1]).

- Yatai et al state that "while 23 enriched genes were reported, it was not verified whether these enriched genes were (up) activated or (down) inactivated". Figure 2C [1] reports $p$ values for "associations between each consensus class and the activity status of 23 regulons", as we described in the Methods section. For example, the PPARG regulon is activated in the luminal subtypes and the FOXM1 regulon in the basal/squamous subtype. Regulon activities have been validated in 17 TCGA (The Cancer Genome Atlas) cohorts [9] and with in vitro knockdown assays [10].

- Yatai et al also state the need to investigate the prognostic value of molecular subtypes, citing work (their reference [5]) that considered what may help surgeons decide between partial and radical cystectomy for patients diagnosed with bladder-adherent colorectal carcinoma. We find that this colorectal cancer citation lacks relevance to our work, which applies to patients with primary urothelial bladder cancer.

- We analyzed treatment response according to the consensus classes in retrospective series, discussed selection of patients for anti-FGFR treatments, and suggested therapeutic targets for each class. We reported biological subtype characteristics that may have clinical value (Fig. $5 C$ [1]). Several publications [6,11,12] reported on the prognostic value of individual subtypes and on their possible predictive value for response to chemotherapy and immunotherapy, or suggested proposed treatments by subtype as a roadmap for clinical testing and validation 
[6]. We found no significant association between subtypes and pathological neoadjuvant chemotherapy (NAC) response (or survival after NAC) in this larger data set, and suggest that future studies investigate this issue in the context of the consensus classes.

We reiterate what we stated in our article [1]: there is an outstanding need, but also an opportunity, for the MIBC community to use and validate these consensus subtypes in completed and ongoing prospective clinical trials.

Conflicts of interest: Roland Seiler and Peter C. Black share a patent with GenomeDx. Peter C. Black has received in-kind research funding from GenomeDx; is an advisory board member for AbbVie, Asieris, AstraZeneca, Astellas, Bayer, Biosyent, BMS, Janssen, Lilly, Merck, Roche, Sanofi, and Urogen; and has participated in clinical trials with Genentech, Janssen, Ferring, Astellas, Sitka, and MDx Health. Joaquim Bellmunt has received research funding from Takeda, Pfizer, Novartis, and Sanofi-Aventis, and has an advisory role for Genentech, MSD, Pfizer, GSK, BMS, Pierre Fabre, Sanofi-Aventis, Astellas, OncoGenex, and Janssen. Lars Dyrskjøt has received research funding from Natera and Ferring, and has an advisory role for Ferring. David J. McConkey has received research funding from AstraZeneca and is a consultant for Rainier Pharmaceuticals. Seth P. Lerner is a consultant for UroGen and Vaxiion; has received support for a clinical trial from Endo, FKD, Urogen, and Viventia; and is an advisory board member for Ferring, miR Scientific, QED Therapeutics, and UroGen. Yves Allory is an advisory board member of AstraZeneca. The remaining authors have nothing to disclose.

\section{References}

[1] Kamoun A, de Reyniès A, Allory Y, et al. A consensus molecular classification of muscle-invasive bladder cancer. European Urology 2020;77:420-33.
[2] Lerner SP, McConkey DJ, Hoadley KA, et al. Bladder cancer molecular taxonomy: summary from a consensus meeting. Bladder Cancer 2016;2:37-47.

[3] Yatai KB, Dunning MJ, Wang D. Consensus genomic subtypes of muscle-invasive bladder cancer: a step in the right direction but still a long way to go. European Urology 2020;77:434-5.

[4] Guinney J, Dienstmann R, Wang X, et al. The consensus molecular subtypes of colorectal cancer. Nat Med 2015;21:1350-6.

[5] Sjödahl G, Eriksson P, Liedberg F, Höglund M. Molecular classification of urothelial carcinoma: global mRNA classification versus tumour-cell phenotype classification. J Pathol 2017;242:113-25.

[6] Robertson AG, Kim J, Al-Ahmadie H, et al. Comprehensive molecular characterization of muscle-invasive bladder cancer. Cell 2017; 171:540-56.

[7] Chen GM, Kannan L, Geistlinger L, et al. Consensus on molecular subtypes of high-grade serous ovarian carcinoma. Clin Cancer Res 2018;24:5037-47.

[8] Rashid NU, Peng XL, Jin C, et al. Purity Independent Subtyping of Tumors (PurIST), a clinically robust, single-sample classifier for tumor subtyping in pancreatic cancer. Clin Cancer Res 2020;26:82-92. http://dx.doi.org/10.1158/1078-0432.CCR-19-1467.

[9] Alvarez MJ, Shen Y, Giorgi FM, et al. Functional characterization of somatic mutations in cancer using network-based inference of protein activity. Nat Genet 2016;48:838-47.

[10] Campbell TM, Castro MAA, de Santiago I, et al. FGFR2 risk SNPs confer breast cancer risk by augmenting oestrogen responsiveness. Carcinogenesis 2016;37:741-50.

[11] Choi W, Porten S, Kim S, et al. Identification of distinct basal and luminal subtypes of muscle-invasive bladder cancer with different sensitivities to frontline chemotherapy. Cancer Cell 2014;25:15265 .

[12] Mariathasan S, Turley SJ, Nickles D, et al. TGF $\beta$ attenuates tumour response to PD-L1 blockade by contributing to exclusion of T cells. Nature 2018;554:544-8. 